\title{
Implementierung dezentraler Produktionskonzepte
}
A. Wandel der Fabrikstrukturen
1. Implementierung dezentraler Produktionskonzepte als Herausforderung
2. Dezentrale Produktionskonzepte als Implementierungsobjekte
3. Implementierungskonzepte als Leitlinien der Implementierungsarbeit

B. Implementierungsbedarfe

C. Implementierungsparameter
1. Information

2. Qualifikation

3. Motivation

4. Projektorganisation

5. Angleichung

D. Implementierungsprozeduren

1. Planungsprozeduren

2. Diffusionsprozeduren

3. Strukturierungsprozeduren

Literatur 


\section{A. Wandel der Fabrikstrukturen}

\section{Implementierung dezentraler Produktionskonzepte als Herausforderung}

Die Implementierung (Einführung, Umsetzung, Realisierung oder auch „Promotion ${ }^{\star}$ ) von dezentralen Produktionskonzepten stellt für das Produktionsmanagement in zweifacher Hinsicht eine Herausforderung dar. Zum einen werden segmentierte, modulare, fokussierte, dedizierte, teamorientierte bzw. fraktale Produktionskonzepte gemeinhin als ein kritischer Erfolgsfaktor einer wettbewerbsfähigen Produktion eingestuft. Die besondere Stärke dieser Produktionskonzepte liegt letztlich darin begründet, daß sie einen hohen Zielerreichungsgrad bei allen Zielen ermöglichen, die üblicherweise durch die Produktion verfolgt werden (vgl. Abbildung 1).

Der Erfolg oder Mißerfolg von Produktionskonzepten wird an zwei ${ }_{n}$ Meßpunkten " gemes: sen, am Grad der Programmerfüllung (Output, Marktanteile, Kundenzufriedenheit usw.) sowie am Grad der Ressourcennutzung (Input, Verfügbarkeit von Leistungspotentialen, Qualifizierung etc.). In den Lehrbüchern zum Produktionsmanagement finden sich zahlreiche Hinweise darauf, daß zwischen diesen beiden Strängen von Erfolgskriterien leicht Zielkonflikte auftreten können. Hierzu zählt unter anderem das Dilemma der Ablaufplanung, sprich der Konflikt zwischen Minimierung der Durchlaufzeiten (Indikator für gute Aufgabenerfüllung) und Maximierung der Nutzungszeiten (Kennzahl für gute Ressourcennutzung). In der aktuellen Segmentierungs- und Teamvon Zielharmonie die Rede: Dezentrale Produktion wird gleichzeitig als marktgerecht (flexible, kundennahe Marktbearbeitung) und als mitarbeitergerecht (hochgradige Ressourcenmobilisierung bei überschaubaren Koordinations- bzw. Transaktionskosten) eingestuft.

Zum anderen besteht Einigkeit darüber, daß der Erfolg neuer Produktionskonzepte nicht in der Entwurfsphase, sondern in der Einführungsphase entschieden wird. Weniger die abstrakten Lemprozesse am Schreibtisch (,Simulieren“) als vielmehr die konkreten Erfahrungsprozesse vor Ort ${ }_{n}$ auf der Baustelle ${ }^{\text {" („Prototyping }}{ }^{4}$, ,Versuch und Irrtum-Lernen ${ }^{\circ}$ ) weisen den Weg zu adäquaten Produktionsstrukturen. Dieser Mangel an „Eleganz ${ }^{\star}$ erklärt sich aus der Radikalität der Umstrukturierung. Mit der Dezentralisation der Produktion erfolgt nicht bloß eine Änderung einer Organisationsform. Vielmehr findet ein Wandel im Organisationsprinzip durch die Umstellung von Fremd- auf Selbstorganisation statt, flankiert durch andere Prinzipien wie beispielsweise Kundenorientierung und ProzeBorientierung. Entsprechend hoch sind auch die Anforderungen an die Professionalität der Umsetzungsprozesse. 


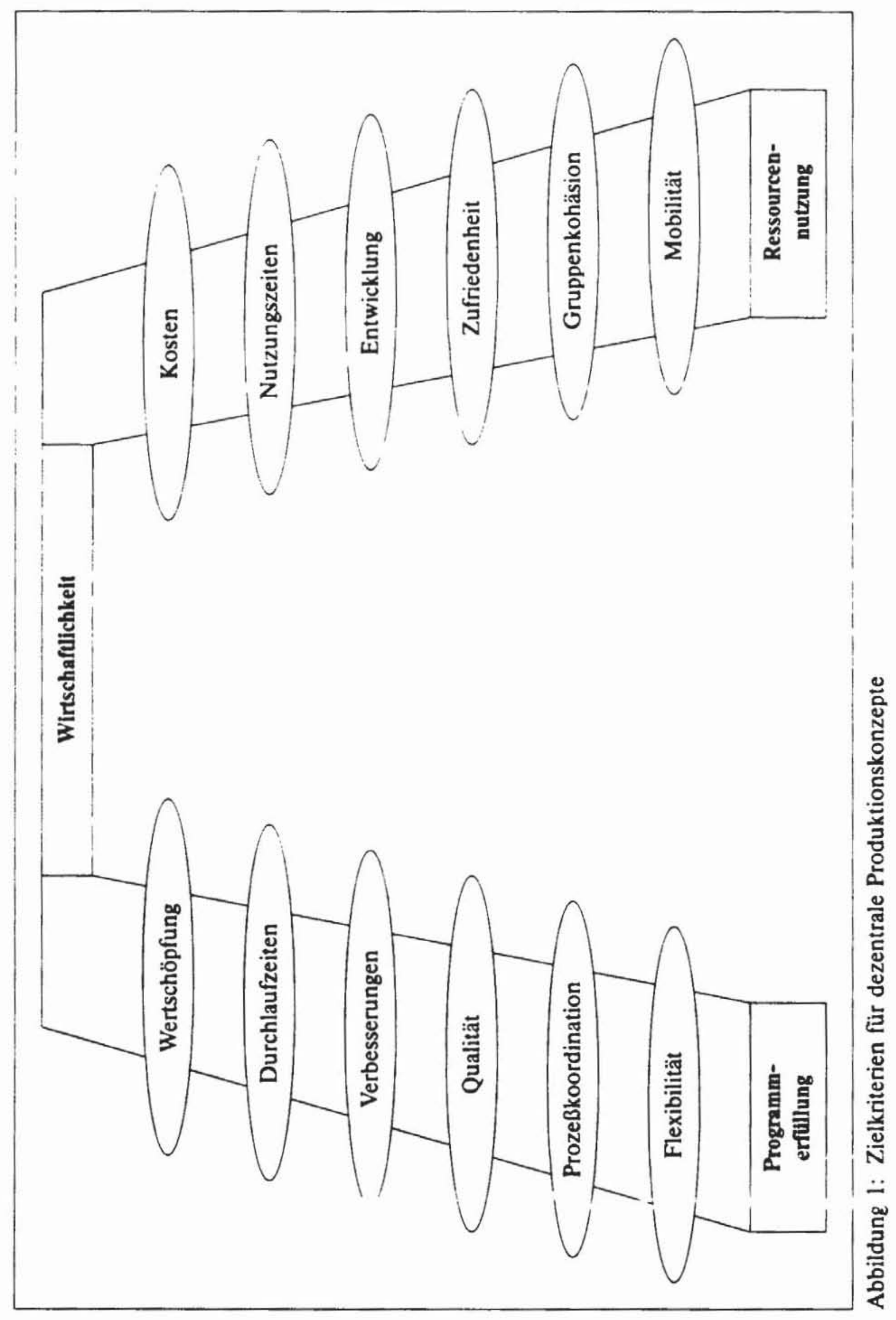




\section{Dezentrale Produktionskonzepte als Implementierungsobjekte}

Die einzuführenden dezentralen Produktionskonzepte lassen sich sowohl nach technischformalen als auch nach betriebswirtschaftlich-inhaltlichen Merkmalen charakterisieren. Formale Beschreibungen stellen auf die überschaubare Größe, die homogene Binnenstruktur (Teilefamilien als Leistungsprogramm, Anwendung des Gruppenprinzips) sowie die geringe Anzahl von Schnittstellen einer dezentral operierenden Produktionseinheit zu den benachbarten Organisationseinheiten ab (Komplettbearbeitung, schnittstellenminimierende Flußoptimierung im Waren- und Informationsfluß). Dieser Sichtweise entsprechen Bezeichnungen wie Segment (i.w.S.), Zelle, Fertigungsbereich, Einheit/Unit (z.B. selbständige Produktionseinheiten), Insel (Fertigungs-, Produktions- bzw. Produktinsel) oder auch teilautonome Teams (Gruppen). Sekundärorganisatorische Konzepte der Zirkel-und Projektarbeit werden dadurch prinzipiell aus dem Spektrum der dezentralen Produktionskonzepte ausgegrenzt. Inhaltliche Kennzeichnungen betonen die unternehmerische Funktionsweise der dezentralen Einheiten im Sinne von „Centern ${ }^{,}$, Unternehmen im Unternehmen ${ }^{4},{ }_{\text {Mini Companies }}$ oder "Business Teams". Differenziert wird nach der Art der übernommenen Verantwortung (für Produktivität, Qualität, Termine, direkte Kosten, Umsätze, Deckungsbeiträge usw.) in Leistungs-Center, Cost-Center, Profit-Center etc. Das Cost-Center stellt die am weitesten verbreitete Center-Variante im Produktionsbereich dar.

Die Gesamtstruktur dezentraler Produktionskonzepte setzt sich aus drei Teilstrukturen zusammen. Die Inter-Center-Strukturen regeln das Zusammenspiel zwischen den einzelnen Segmenten über die Wertschöpfungskette (horizontaler Verbund) sowie zwischen Cost-Centern und übergeordneten Profit-Centern (vertikaler Verbund).

Die Extra-Center-Strukturen decken die Außenbeziehungen zwischen den Segmenten und allen anderen Steuerungseinrichtungen bzw. Organisationseinheiten ab, die selbst nicht als Center organisiert sind. Dazu zählt neben der Einbindung der Center in eine zentrale PPS vor allem die Schnittstelle zwischen Centern und Zentralbereichen, also den Querschnitts- bzw. Service-Funktionen in Form von Einkauf, Vertrieb, Konstruktion, Qualitätssicherung, Logistik, Werkzeugwesen, Arbeitsvorbereitung, Instandhaltung, Disposition usw.

Die Intra-Center-Strukturen werden durch die Parameter zur Gestaltung der Binnenstruktur eines einzelnen Segments bestimmt (vgl. Abbildung 2).

Die Gestaltungsparameter erster Ordnung umfassen die Gestaltung des Segmentprogramms (z.B. Leistungsbreite- und tiefe, interne und externe Kunden), der Segmentressourcen (Maschinengruppen, Humanressourcen, Gebäude usw.) sowie der internen Segmentorganisation. Die Gestaltungsparameter zweiter Ordnung handhaben die Schnittstellen zwischen diesen drei Sektoren, etwa die programmgerechte Bemessung der Ressourcen oder die Abklärung von Enţ̧ elt fragen (beispielsweise anforderungs- und leistungsgerechte Entlohnung).

Nur über eine simultane Gestaltung aller drei Teilstrukturen kann der optimale Grad der Autonomie und Autarkie eines Centers festgelegt werden. Der Autarkiegrad ergibt sich aus 


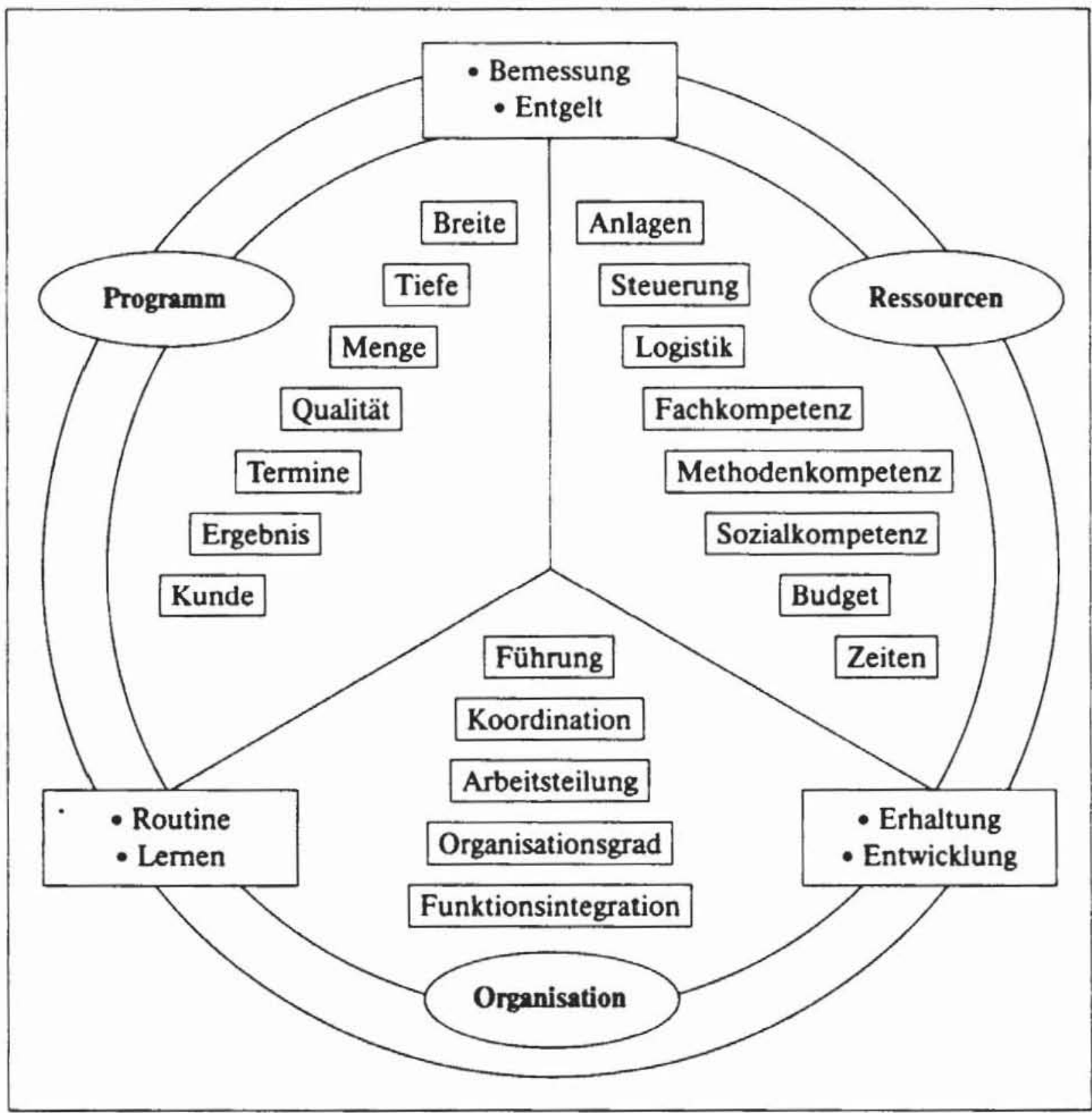

Abbildung 2: Parameter der Center-Gestaltung

der Integration von Primär- und Sekundärfunktionen (Leistungstiefe) sowie der dazu erforderlichen Ressourcen in das einzelne Center.

\section{Implementierungskonzepte als Leitlinien der Implementierungsarbeit}

Implementierungsarbeit ist an der Schnittstelle zwischen Produktionskonzept und Produktionskontext zu leisten. Sie bezweckt die Herstellung eines Fit zwischen den zu implementierenden Produktionsformen und den vorfindlichen Gegebenheiten, in welche die neuen Konzepte eingepflanzt werden sollen. Hierzu zählen unter anderem der Altbestand an Organisationsstrukturen, die Vorstellungen aller Betroffenen von einer akzeptablen Produktionsstruktur, die Rahmenbedingungen in den angrenzenden Funktionsbereichen 


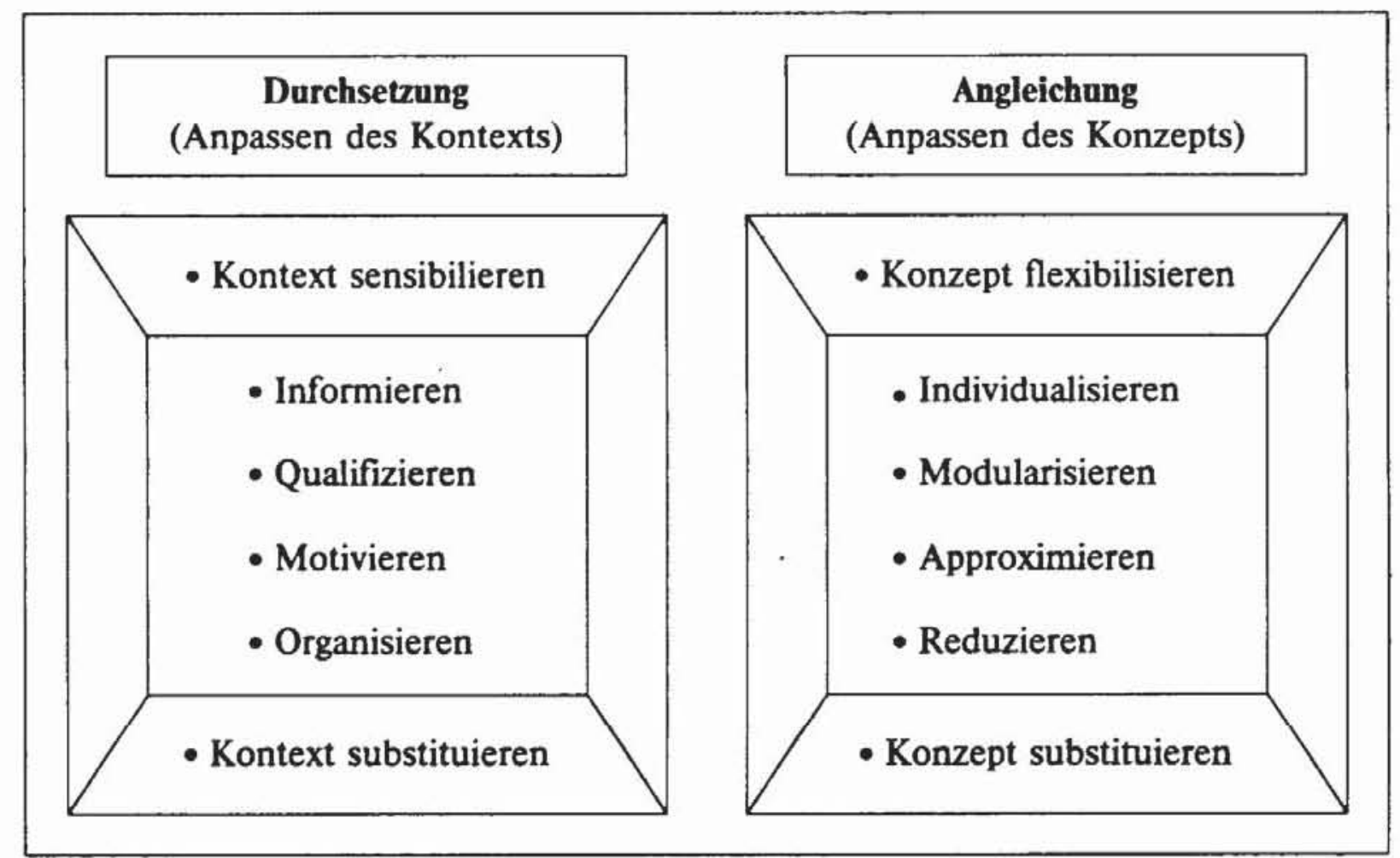

Abbildung 3: Instrumentarium der Implementierung

sowie unternehmungsexterne Kontextfaktoren bei Zuliefer- und Abnehmerunternehmen.

Implementierungsaktivitäten starten mit der Diagnose des vorhandenen ${ }_{n}$ Misfits ${ }^{\star}$ Zwischen Konzept und Kontext. Im Verlauf des Implementierungsprozesses wird der Erfolg der Implementierungsbemühungen anhand von geeigneten "harten " bzw. "weichen" Erfolgskennzahlen gemessen (Durchlaufzeiten, Zufriedenheit der Mitarbeiter sowie internen und externen Kunden). Auf diesem Wege können sich die Implementierungsverantwortlichen jeweils ein Bild vom aktuell herrschenden Implementierungsbedarf machen.

Alle Interventionsmaßnahmen sollen den ermittelten Implementierungsbedarf decken (vgl. Abbildung 3). Dies kann naturgemäß entweder durch Anpassung des Kontexts an das Produktionskonzept (Durchsetzung) oder umgekehrt durch Anpassung des Konzepts an den Kontext (Angleichung) vonstatten gehen.

\section{B. Implementierungsbedarfe}

Restrukturierungsbedarfe in der Produktion lassen sich nur sehr rudimentär durch einmalige Diagnose-Operationen ermitteln. Da sowohl die Produktionskonzepte als auch der Produktionskontext im Zeitablauf exogenen und endogenen Veränderungen unterliegen (z.B. steigende Anforderungen, neue Mitarbeiter und Zulieferer), erfordert professionelle Implementierung ein permanentes „Scanning“. 
Die Größe des Implementierungsbedarfs hängt zunächst von der Diskrepanz zwischen alten und neuen Produktionskonzepten ab. Liegt beispielsweise eine ausgeprägte verrichtungsorientierte Werkstattfertigung mit zentraler Planung der Produktion, Qualitätssicherung, Lagerung usw. vor, dann ist der Weg zu einer dezentral-segmentierten Produktionsstruktur infolge des zu überwindenden Fragmentierungs- und Zentralisationsgrades recht weit.

Als zweite Determinante geht die vertikale Segmentierungstiefe in die Berechnung des Implementierungsbedarfs ein. Nur in den seltensten Fällen ist die Dezentralisation der Produktion auf eine Ebene beschränkt. In aller Regel sind mehrere Ebene im Rahmen einer mehrstufigen Segmentierung betroffen (vgl. Abbildung 4). Dazu zählen in der Produktion selbst die Standort- bzw. Werksebene, die Ebene der Cost-Center bzw. Fertigungssegmente i.e.S., die Insel- und die Teamebene.

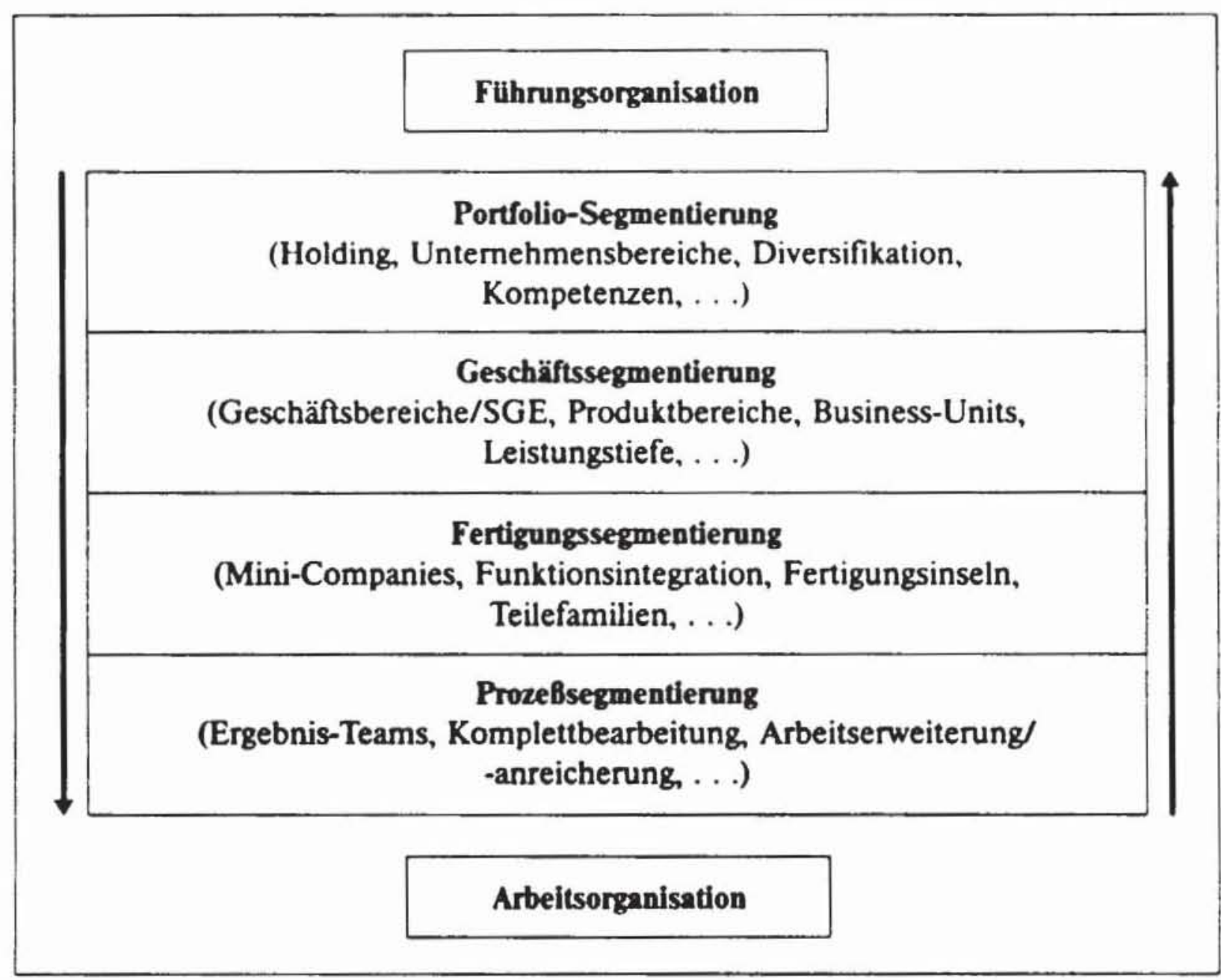

Abbildung 4: Mehrstufige Segmentierung

GroB angelegte Dezentralisationsvorhaben erstrecken sich mitunter auch auf die Geschäftssegmentierung sowie die Anpassung der Leistungstiefe durch Outsourcing (vgl. den Beitrag von Rommel in diesem Band). Dabei wird ja nichts anderes als eine unternehmensübergreifende Dezentralisation der Produktion vorgenommen. 
Der Implementierungsbedarf wird außerdem durch die Breite der in Angriff genommenen

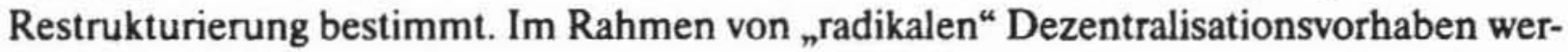
den sowohl die direkten Leistungsbereiche als auch die Dienstleistungsbereiche als Center organisiert. Für eine Beibehaltung der Zentralbereiche (als Service-Center) spricht nicht zuletzt, daß manche Leistungscenter vom Geschäftsvolumen so klein sind, daß sie eigene ${ }_{n}$ Gemeinkostenbereiche“ nicht tragen können.

\section{Implementierungsparameter}

\section{Information}

Ohne eine rechtzeitige Information der Betroffenen ist jedes Dezentralisationsvorhaben zum Scheitern verurteilt. Der Kreis der Betroffenen erweist sich bei Dezentralisationsprojekten als recht groß. Er umfaßt neben den Mitarbeitern, Meistern, Arbeitnehmervertretern und Führungskräften in der Produktion auch die benachbarten Funktionsbereiche, externe Kunden und Lieferanten und schließlich - mit Blick auf wettbewerbliche und beschäftigungspolitische Relevanz der Umstrukturierung - auch die Öffentlichkeit. Ein erster Grundsatz der Informationspolitik betrifft die differenzierte Ansprache der einzelnen Zielgruppen mit unterschiedlichen Medien, Informationsveranstaltungen und teilweise auch zu unterschiedlichen Zeitpunkten, meist in einer Top down-Abfolge über die betroffenen Hierarchie-Ebenen. Besonderes Augenmerk ist auf eine ausgewogene Argumentation zur Begründung der Dezentralisationsabsichten zu richten. Neben den Vorteilen der Dezentralisation (kürzere Wege, Enthierarchisierung, Abspecken der Gemeinkostenbereiche usw.) sollte man auch über die Segmentierungskosten informieren, also über die Kosten der Autarkisierung der dezentralen Bereiche im Zuge der Funktionsintegration. Im Rahmen einer rational durchgeführten Aufklärungsarbeit gilt es auch nachzuweisen, $\mathrm{da} B$ nkleinere" Lösungen für eine kundennahe Produktion, etwa die Einrichtung von Auftragszentren, nicht die erforderliche Effizienzsteigerung erbringen würden.

\section{Qualifikation}

Im Mittelpunkt der Trainingsprogramme steht die „Umschulung “ der Meister zu „Unternehmern". Dabei sind alle Kompetenzsparten abzudecken. Im Center ist für die entsprechende Breite des fachlichen Kompetenzspektrums zu sorgen. Meist werden hier mehrere, unterschiedlich breite Funktionsbilder zugrundegelegt, von denen nur eines dem Ideal des ${ }_{n}$ Allrounders ${ }^{\mu}$ entspricht. Erfolgsentscheidend ist darüber hinaus die Vermittlung eines signifikant erweiterten Zusammenhangsverständnisses. Methodenkompetenz und Sozialkompetenz bilden die unverzichtbare Voraussetzung für die Bewältigung der gestiegenen Autonomie. Diese bringt ja auch neue Spielregeln für den Umgang zwischen den Kunden- und Lieferanten bzw. - besonders bei Installation funktionsgleicher, redundanter 
Segmente an unterschiedlichen Standorten - zwischen Konkurrenten in einem dezentralen Produktionssystem mit sich.

\section{Motivation}

Für die Übernahme von Verantwortung durch die "Mit-Unternehmer" muß geworben werden. Hierbei kann man sich die positiven Anreizeffekte von erweiterten und angereicherten Tätigkeitsfeldern sowie das Motivationspotential von Partizipation im Umstellungsproze $B$ zunutze machen. Die Verlierer der Neustrukturierung, allen voran die in den Zentralbereichen überflüssig gewordenen Spezialisten sowie alle Führungskräfte, die Verantwortung abgeben, müssen entschädigt werden. Vorübergehend sind eventuelle Einkommensverluste durch Umstellung des Entgeltsystems auszugleichen (Besitzstandwahrung).

\section{Projektorganisation}

Die Spezifika der Aufbauorganisation für Dezentralisationsprojekte in der Produktion betreffen folgende Bausteine (vgl. Abbildung 5). Zunächst muß sichergestellt werden, daß in der Kernprojektgruppe alle betroffenen Fach- und Stabsabteilungen unter einer unvoreingenommenen Projektleitung versammelt sind. Desweiteren werden Koordinationsgremien besonders dann erforderlich, wenn die Restrukturierung mehrere Segmentierungsebenen abdeckt (vgl. Abbildung 4). Schließlich ergibt sich umso mehr Bedarf an unternehmungsexterner Unterstützung (durch Beratungsunternehmen, Forschungseinrichtungen usw.), je konfliktbeladener und innovativer die anstehende Restrukturierung ist.

Üblicherweise wird in der Ablauforganisation der Projekte eine Phaseneinteilung in eine Pilotphase und eine Konsolidierungsphase vorgenommen. Als Erfolgsfaktoren für die Bestimmung der Pilotbereiche sind neben der Größe dieser Experimentierfelder vor allem deren „Dezentralisationsfähigkeit ${ }^{\text { }} \mathrm{zu}$ beachten. Hier werden bevorzugt Bereiche ausgewählt, die sich schnell und ohne große Widerstände umstrukturieren lassen, so daß die Projektverantwortlichen möglichst prompt mit Erfolgsmeldungen aufwarten können. Im Normalfall strebt man mit der Konsolidierung eine flächendeckende Diffusion der dezentralen Strukturen über den gesamten Produktionsbereich an. Die Flächendeckung selbst geht entweder stufenweise oder „auf einen Schlag“ vonstatten.

\section{Angleichung}

Beim Entwurf eines geeigneten Konzepts für eine dezentrale Produktion können sich Produktionsmanager entweder an bereits praktizierten Vorbildern oder an „vorgefertigten “ Standard-Konzepten, etwa für die Grobstruktur einer Fertigungs- bzw. Produktinsel, orientieren. Im Einführungsprozeß müssen an dem ausgewählten Ausgangskonzept je- 


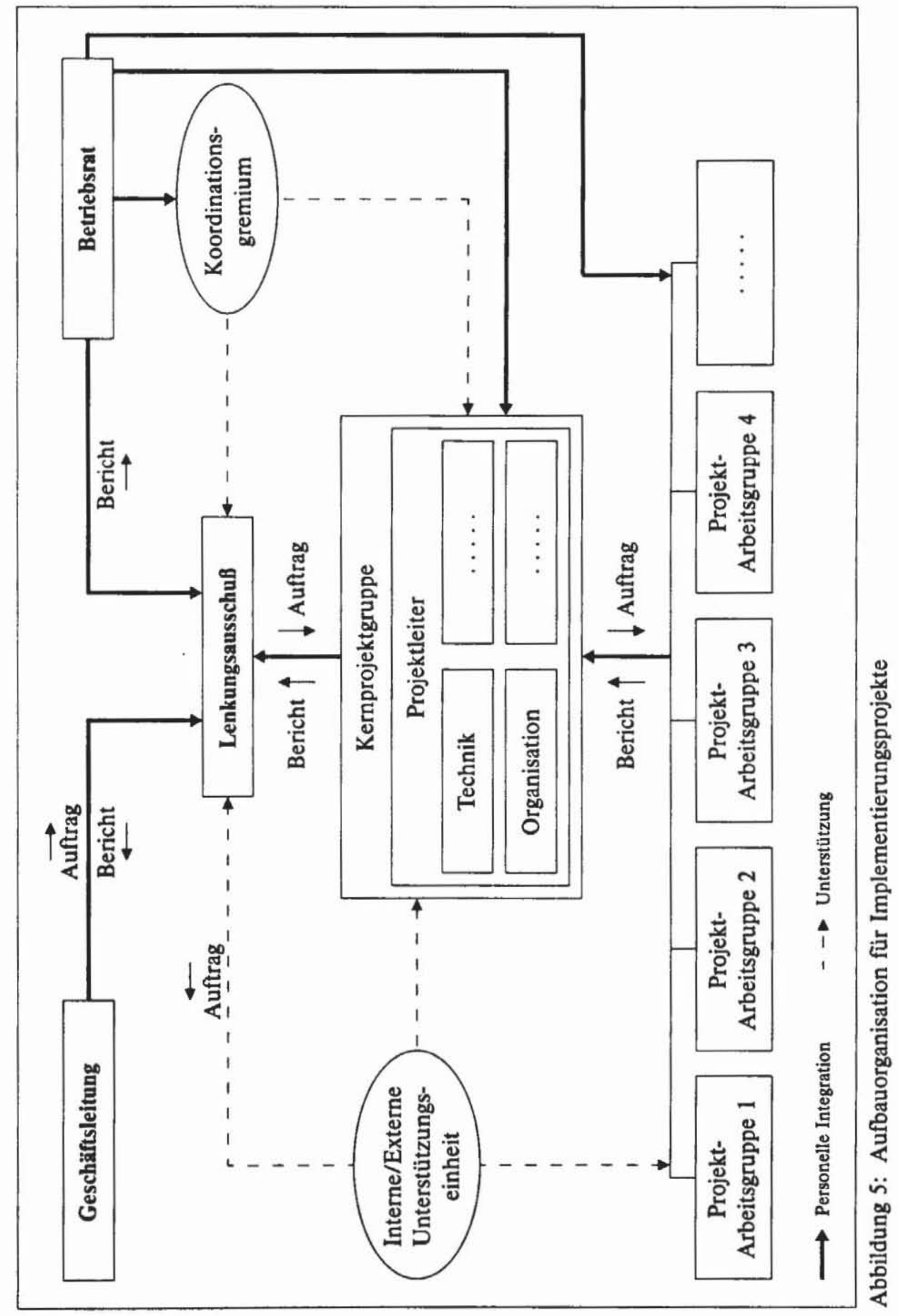


doch meist größere Modifikationen vorgenommen werden. Hierzu zählt durchweg das kontextspezifische Customizing (statt Kopieren) von Vorbildern und Konzepten "von der Stange “. Außerdem werden viele Konzepte nicht als Ganzes, sondern Baustein für Baustein eingeführt (z.B. zuerst Center-Spezifikation, dann Schulung, dann Entlohnungsmodell). $\mathrm{Zu}$ diesem $\mathrm{Zweck}$ muß das Gesamtkonzept in geeignete Module zerlegt werden. Im Zuge der Implementierung werden oft provisorische Zwischenlösungen installiert. Bevor beispielsweise die Verantwortung für Qualität, Nutzungsgrade, Termine und bestimmte Kostenarten auf teilautonome Teams übertragen werden kann, müssen die Grundregeln der solidarischen Zusammenarbeit sowie der kollektiven Wahrnehmung solcher Pflichten im Rahmen von Qualitätszirkeln oder Projektgruppen geübt werden.

\section{Implementierungsprozeduren}

\section{Planungsprozeduren}

Auch bei der Planung von komplexen Restrukturierungsvorhaben bewähren sich die allgemeinen Vorgehensweisen einer koordinierten und flexiblen Planung. Mit Hilfe des Gegenstrom-Prinzips kann man den Spezifika des Einführungsprozesses für neue Produktionskonzepte auf Unternehmens- und Werksebene Rechnung tragen (vgl. Abbildung 6).

Durch das Gegenstrom-Prinzip läßt sich zum einen verhindern, daß allen Werken schematisch dasselbe Produktionskonzept übergestülpt wird. Funktionstüchtige Produktionsstrukturen variieren von Werk zu Werk mit den werksspezifischen Kontextfaktoren (große versus kleine Standorte, junge versus alte Standorte usw.). Innerhalb eines Standorts muB von Fertigungsbereich zu Fertigungsbereich für Variabilität gesorgt werden. Das betrift etwa die Frage, inwieweit in Abhängigkeit vom Anspruchsniveau der Wartungs- und Instandsetzungsaufgaben nach dem TPM-Konzept („Total Productive Maintenance“) die anfallenden Instandsetzungsaufgaben in eine Fertigungsinsel verlagert werden können.

Zum anderen kann man durch das Gegenstrom-Verfahren in der Einführungsplanung sicherstellen, $\mathrm{da} B$ die (begrüßenswerten) Einzelinitiativen an der Basis registriert und honoriert werden. Im Bottom up-Proze $B$ der Einführungsplanung ist abzuklären, inwieweit diese Konzepte möglicherweise mit einer gewissen Vorbildfunktion in die Gestaltung eines Rahmenkonzepts Eingang finden können.

\section{Diffusionsprozeduren}

In der Konsolidierungsphase ist neben dem Diffusionstempo auch festzulegen, welche konkreten Produktionsbereiche in welcher Reihenfolge in das dezentrale Produktionskonzept integriert werden sollen. In aller Regel werden zunächst die (end-)kundennahen Montagebereiche (Montageinseln) autonomisiert und autarkisiert. Im AnschluB daran 


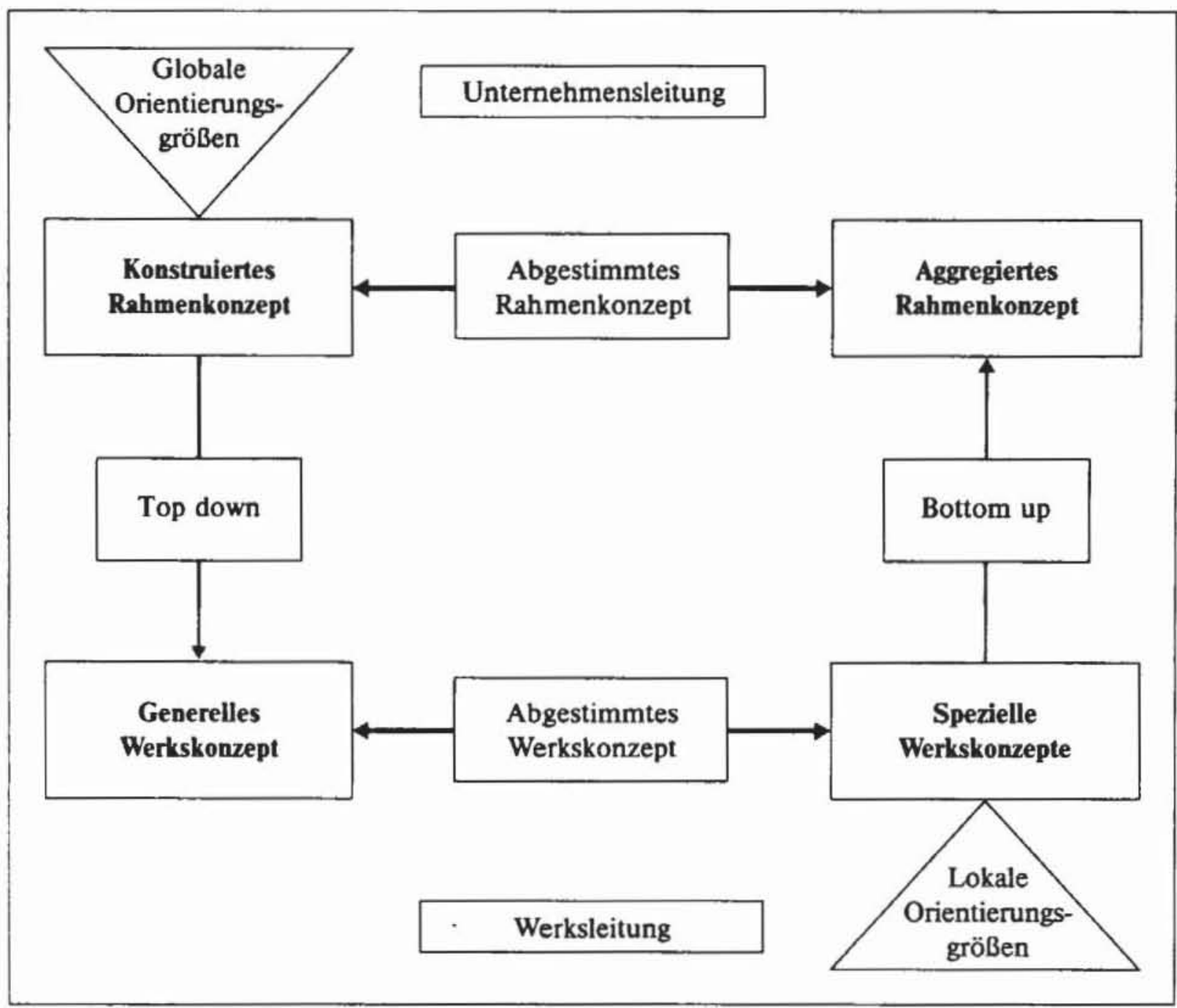

Abbildung 6: Gegenstrom-Implementierung

werden die vorgelagerten Produktionsstufen der Aggregate- bzw. Komponentenfertigung (Fertigungsinseln i.e.S.) zu Cost-Centern ausgebaut. Vorübergehend kommt es mitunter zur Installation von provisorischen Konstruktionsinseln bzw. Planungsinseln (Arbeitsvorbereitung). Bei den meisten Diffusionsprozeduren bildet die Integration der ServiceBereiche als Dienstleistungscenter den Schlußstein des Dezentralisationsprojekts. Im Idealfall entscheiden die zuvor installierten Leistungszentren als ${ }_{n}$ Kunden “ dieser ServiceEinheiten, welches Dienstleistungsspektrum von den Service-Zentren angeboten (inhouse outsourcing, Rumpfsteuerung) und welche indirekte Funktionen im Leistungscenter selbst integriert werden sollen.

Auf dem Gebiet der hierarchisch-mehrstufigen Restrukturierung (vgl. Abbildung 4) läßt sich feststellen, daß Dezentralisationsprojekte parallel auf mehreren Ebenen initialisiert werden. Für einige Unternehmen stellt die Geschäftssegmentierung, für andere die Teamarbeit, die aearzerten Staudorte uder die Fertigungssegmentierung den Einstieg in die Dezentralisation dar. Als erste Etappe im Rahmen aller Dezentralisationsvorhaben sollte eine Prozeßanalyse der „Operations“ (Flußoptimierung) und der Geschäftsprozesse erfolgen. 


\section{Strukturierungsprozeduren}

Beim Aufbau eines umfassenden Produktionskonzepts (vgl. Abschnitt A.2.) muB zunächst die Reihenfolge bestimmt werden, in der man sich um die Neustrukturierung der Binnenstruktur eines Centers, des Inter-Center-Verbunds und der Beziehungen zwischen Center-Organisation einerseits und Zentralbereichen andererseits kümmert.

Bei der internen Strukturierung eines autonomen und autarken Segments bzw. Centers ist prozedural festzulegen, in welcher Abfolge die einzelnen Parameter spezifiziert werden (vgl. Abbildung 2). Herkömmliche Faustregeln wie beispielsweise „Strategie vor Technik vor Organisation vor Personal" haben hier ihre Allgemeingültigkeit verloren. Mitunter lösen architektonische Umbaumaßnahmen mehr oder weniger zufällig ein Dezentralisationsprojekt aus. Oft hinkt die Anpassung des Steuenungsprinzips (z.B. Umstellung von Pull- auf Push-Steuerung in der Materialwirtschaft, Steuerung durch kaufmännisches Controlling) oder der Steuerungstechnik (dezentrale PPS, Leitstandkonzepte usw.) mit deutlicher Verzögerung hinter der Anpassung der anderen Bausteine hinterher. 


\section{Literatur}

Antoni, C., Eyer, E.: Fertigungsinsein und Entgelt, in: Personal, 45. Jg. (1993), S. 108-114.

AWf (Ausschuss fur wirtschaftliche Fertigung E.V.) (Hrsg.): Fertigungsinseln, Bad Soden 1988.

AWF (Ausschuss fur wirtschaftliche Fertigung E.V.) (Hrsg.): Integrierte Fertigung von Teilefamilien, Köln 1990.

Bонr, K.; Eberwein, R.-D.: Die Organisationsform Fertigungsinsel, in: WiSt, 18. Jg. (1989), S. 218-223.

BÜнNer, R.: Arbeitsstrukturierung und Personaleinsatz in Fertigungsinseln, in: WISU, 15. Jg. (1986), S. $493-497$. BUHNER, R - Betriebswirtschaftliche Organisatıonslehre, 6. Aufl., München/Wien 1992.

Clauss, M.: Die Strategie der Implementierung in der Unternehmung, Pfaffenweiler 1989.

CoRsten, H.; WILL, T: Strategieunterstützung durch Fertigungsegmentierung - Möglichkeiten und Grenzen, in: WISU, 21. Jg. (1992), S. 397-402.

Eversheim, W., Cremer, R.; Schneewind, J.: Aufgabenintegration bei der Produkt- und ProzeBgestaltung, in: Aufgaben- und Rechnerintegration - ein Gegensatz zur schlanken Produktion?, hrsg. v. VDI, Düsseldorf 1992, S. $125-147$.

Horn, V.: Truge, P.: Segmentierung steigert die Leistung, in: ZwF, 87. Jg. (1992), S. 309-312.

KALuza, B.: Betriebswirtschaftliche und fertigungstechnische Aspekte der Gruppentechnologie, in: CIM Management, 8. Jg. (1992), H. 6, S. 16-23.

MARr, R.; Kötring, M.: Implementierung, organisatorische, in: HWO, hrsg. v. E. Frese, 3.Aufl., Stuttgart 1992, Sp. 827-841.

Nitᄂ. B.: Marktorientierte Neustrukturierung eines Maschinenbau- und Fahrzeugunternehmens, in: Lean Management. Der Weg zur schlanken Fabrik, hrsg. v. H. Wildemann, München 1992, S. 51-76.

Nutr, P. C.: Tactics of Implementation, in: Academy of Management Journal, 29. Jg. (1986), S. 230-261.

Pieper, A.; Strotgen, J.: Produktıve Arbeitsorganisation: Handbuch für die Betriebspraxis, Köln 1990.

Reiss, M.; Corsten, H.: Grundformen der Produktionsorganisation, in: WISU, 19. Jg. (1990), H. 4, Studienblatt.

SChulte, C: Das Modell der Fertigungssegmentierung aus personeller und organisatorischer Sicht, Bergisch Gladbach/Köln 1989.

Singl, G.: Kundenorientierte Produktion in dezentralen Organisationseinheiten - Vom Ansatz zur Erfahrung, in: Kundenorientierte Produktion, hrsg. v. H.-J. Bullinger, Berlin 1992, S. 135-187.

Suzaxi, K.: The New Shop Floor Management, New York/Toronto 1992.

Tress, D.: Kleine Einheiten in der Produktion, in: ZfO, 55. Jg. (1986), S. 181-186.

WAGner, D.: Die Produktinsel, Köln 1991.

WARNECKE, H.-J.: Die Fraktale Fabnk. Revolution der Unternehmenskultur, Berlin 1992.

WildemanN, H.: Einführungsstrategien für eine Just-In-Time-Produktion und-Logistik, in: ZfB, 61. Jg. (1991), S. 149-169.

Wildemann, H.: Die modulare Fabrik. Kundennahe Produktion durch Fertigungssegmentierung, 3. Aufl., St. Gallen 1992.

WILDEMANN, H.: Gestaltungsaspekte indirekter Funktionen in Fertigungssegmenten: Die Bestimmung des Autonomiegrades, in: DBW, 52. Jg. (1992), S. 777-801.

Young, S. M.: A Framework for Successful Adoption and Performance of Japanese Manufacturing Practices in the United States, in: Academy of Management Review, 17. Jg. (1992), S. 677-700.

ZiPfel, G.: Dezentrale PPS-Systeme - Konzepte und theoretische Fundierung, in: Neuere Konzepte der Produktionsplanung und -steuerung, hrsg. v. G. Zäpfel, Linz 1989, S. 29-59. 